\title{
Casos clínicos: ecocardiografía transesofágica y monitorización hemodinámica en cirugía no cardiaca
}

\author{
VERÓNICA MERTZ K., MARIO CONCHA P.
}

\section{Trans-esophageal echocardiography in non-cardiac surgery. Report of four illustrating cases}

Transesophageal echocardiography can assess ventricular filling and contraction and detect motility disturbances secondary to ischemia. In non-cardiac surgery it can be extremely helpful to monitor complex patients. We report a 69-year-old male subjected to a hepatic lobectomy, a 59-year-old male with hepatorenal syndrome, a 52-year-old female subjected to a gastric bypass, and a 54-year-old male subjected to a thyroidectomy. In these four cases, left ventricular motility and preload were evaluated with transesophageal echocardiography, and it was of great aid in decisionmaking during anesthesia.

(Rev Med Chile 2011; 139: 353-356).

Key words: Anesthesia, general; Echocardiography, transesophageal; General surgery.

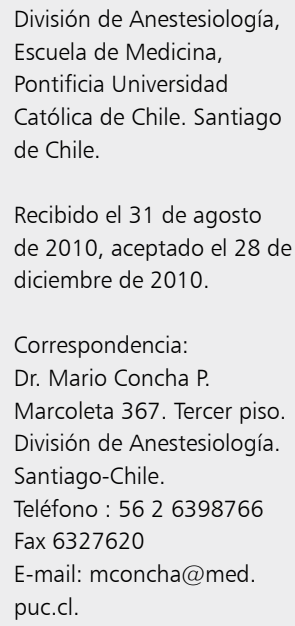

$\mathrm{E}$ l uso de ecocardiografía transesofágica (ETE) ha sido ampliamente recomendado en cirugía cardiaca y de aorta torácica ${ }^{1}$. En cirugía no cardiaca su uso es limitado, existiendo consenso de su utilidad en situaciones de compromiso hemodinámico severo cuyo origen no es posible identificar con los medios diagnósticos habituales ${ }^{2}$. Probablemente una mayor utilización haría evidente los beneficios en otras situaciones. Desafortunadamente, la disponibilidad de ecocardiógrafo, y de un operador entrenado limitan su utilización fuera del ámbito de la cirugía cardiaca. Reportamos cuatro pacientes sometidos a cirugía no cardiaca, en los cuales la ETE entregó información no detectable por otros medios, y que fue considerada relevante para una favorable evolución.

\section{Caso 1}

Paciente de 69 años, en que se realizó lobectomía hepática. Se monitorizó con electrocardiografía, línea arterial, presión venosa central (PVC), sonda urinaria y ETE. La cirugía se desarrolló sin incidentes. Terminada la resección y con hemostasia satisfactoria, presentó progresiva disminución de la presión arterial (PA) hasta $50 \mathrm{mmHg}$ de presión sistólica, con frecuencia cardiaca (FC) 80 por minuto, PVC $4 \mathrm{mmHg}$, Sat $\mathrm{O}_{2} 99 \%$, y $\mathrm{CO}_{2}$ espirado 30. La ETE mostraba llenado y motilidad ventricular izquierda normales y no se visualizó paso de material embólico a través de cavidades derechas o evidencias de sobrecarga de estas. Se postuló una anafilaxis, iniciándose tratamiento con $300 \mathrm{mg}$ de hidrocortisona, aporte de volumen, adrenalina 0,25 y $0,5 \mathrm{mg}$ endovenoso, y luego una infusión hasta $0,3 \mu \mathrm{g} / \mathrm{kg} / \mathrm{min}$, sin obtener efectos sobre PA y FC. Se agregó noradrenalina hasta 3 $\mu \mathrm{g} / \mathrm{kg} / \mathrm{min}$, sin lograr respuesta. Simultáneamente se instaló catéter de arteria pulmonar (CAP) que mostró una presión de arteria pulmonar (PAP) de 22/10 mmHg, presión de capilar pulmonar (PCP) $8 \mathrm{mmHg}$, índice cardiaco (IC) $5,4 \mathrm{~L} / \mathrm{m}^{2}$ y resistencia vascular sistémica indexada (RVSI) de 155 dinas $^{\star}$ segundo $/ \mathrm{cm}^{5} / \mathrm{m}^{2}$. Terminada la cirugía se 
trasladó a tratamiento intensivo donde se apreció gradual recuperación de PA y disminución de los requerimientos de neosinefrina y adrenalina. Se extubó a las 4 horas sin problemas.

\section{Caso 2}

Paciente de 59 años, candidato a trasplante hepático. Presentó un síndrome hepatorenal que motivó un importante aporte de volumen y furosemida, sin obtener respuesta diurética. A las 24 horas de esto se encontró donante. En ese momento el paciente estaba soporoso, con escasa respuesta a estímulos verbales, PA 110/60, FC 115 por minuto, frecuencia respiratoria $35 x^{\prime}$. Un ecocardiograma de superficie mostró buena función ventricular global, dilatación de las cuatro cavidades y presión sistólica de arteria pulmonar (PSAP) de $50 \mathrm{mmHg}$. En pabellón, se intubó e instaló sonda para ETE y CAP que mostró una PVC de $25 \mathrm{mmHg}$, PCP $26 \mathrm{~mm} \mathrm{Hg}$, RVSI de 987 dinas ${ }^{\star}$ segundo/ $/ \mathrm{cm}^{5} / \mathrm{m}^{2}$. El IC y PSAP medidas por CAP y ETE fueron respectivamente 4,7 y 5,3 $1 / \mathrm{m}^{2}$ y $49 / 32$ y 48 . El volumen de fin de diástole del ventrículo izquierdo indexado (VFDVII) fue 98 $\mathrm{ml} / \mathrm{m}^{2}$ (valor normal: $35-75 \mathrm{ml} / \mathrm{m}^{2}$ ). Se aportaron fluidos de manera muy restringida, observándose durante todo el procedimiento buena concordancia entre los valores de IC y PSAP medidos con CAP y ETE. Al término de la cirugía la PVC era $14 \mathrm{mmHg}$, PCP $19 \mathrm{mmHg}$, PAP 33/22 e IC 5,1/ $\mathrm{m}^{2}$. El ETE mostraba una PSAP de 32, IC de $51 /$ $\mathrm{m}^{2}$, y VFDVI $67 \mathrm{ml} / \mathrm{m}^{2}$.

\section{Caso 3}

Paciente de 52 años, hipertensa, obesa mórbida, planificada para by pass gástrico. Durante la inducción anestésica, sin haber modificaciones de PA o FC, presentó alteración del segmento ST sugerente de isquemia. La ETE no mostró alteraciones de la motilidad ventricular global ni segmentaria. Se decide realizar la cirugía, la cual transcurre sin incidentes. Post operatorio sin problemas, dada de alta al tercer día.

\section{Caso 4}

Paciente de 54 años, hipertenso y tabáquico, programado para tiroidectomía total. Inducción anestésica sin alteraciones hemodinámicas, pero con modificación de onda $\mathrm{T}$ y segmento ST sugerente de isquemia. La ETE no mostraba alteraciones de la motilidad ventricular. Se decide suspender y realizar coronariografía que fue normal.

\section{Discusión}

La recomendación del American College of Cardiology y de la American Heart Association, referentes a la utilización de la ETE en cirugía no cardiaca, dice que hay una indicación tipo IIa (Beneficio $>>$ Riesgo) siendo razonable utilizarla en situaciones de alteración hemodinámica severa, persistente y de causa no precisada. Aun cuando el nivel de evidencia de esta recomendación es C, es decir basada en una experiencia limitada, no se trata de una recomendación débil, dado el alto grado de consenso clínico al respecto ${ }^{2}$. En nuestra práctica, además de utilizarla en esta situación, la incorporamos como monitorización de pacientes con patología cardiaca severa, o de cirugía en la que se esperan cambios importantes del volumen intravascular. Esto es concordante con la reciente actualización de las recomendaciones ${ }^{1}$.

En el intraoperatorio, la evaluación del llenado y función ventricular, y el diagnóstico de isquemia miocárdica, pueden ser erróneamente interpretados o no detectados con la monitorización habitual. Los casos reportados ejemplifican estas situaciones. En todos, la información obtenida fue considerada importante para la toma de decisiones, permitiendo definir conductas que, a excepción del paciente del caso 4, resultaron adecuadas, evitando medidas terapéuticas innecesarias, o la realización de exámenes caros y no exentos de riesgo.

La hipovolemia como causa de compromiso hemodinámico durante cirugía intraabdominal no es siempre fácil de descartar. La medición de las presiones de llenado cardiaco habitualmente utilizadas para guiar la reposición de volumen, puede verse afectada por alteraciones de la distensibilidad de la pared ventricular, aumentos de la presión intratorácica o disfunción ventricular, factores que limitan y cuestionan la utilidad de estas mediciones ${ }^{3-10}$. En el paciente del caso $1, y$ en el contexto de una resección hepática, es planteable el desarrollo de hipovolemia que pudiera haber sido insuficientemente corregida. Otras posibilidades diagnósticas que expliquen el deterioro hemo- 
dinámico observado son disfunción miocárdica y embolia pulmonar, esta última poco probable dado el resto de los hallazgos. Ninguna de estas posibilidades es descartable con seguridad con la monitorización habitual. La visualización de condiciones normales de llenado y contracción ventricular, la ausencia de signos de sobrecarga o dilatación de cavidades derechas, y la no visualización de fenómenos embólicos, permite eliminar estas posibilidades, y reafirma la hipótesis de una severa disminución de RVSI, que fue confirmada por el CAP. Los hallazgos ecocardiográficos mencionados permitieron mantener e intensificar una terapia dirigida a aumentar la RVSI, evitar aportes excesivos de volumen frente a un cuadro de hipovolemia relativa, y evitó realizar otros exámenes diagnósticos, que considerando la evolución no aportaban a la solución del problema. En el caso 2 , si bien las presiones de llenado sugieren un volumen ventricular aumentado, la magnitud de la sobrecarga circulatoria pulmonar, la posibilidad de cambios rápidos e importantes del volumen intravascular, y principalmente la posibilidad de guiar la reposición de volumen justifican la utilización de la ETE. La posibilidad de la presencia de várices esofágicas no contraindica el uso de la ETE en trasplante hepático, habiendo sido incorporada como monitorización habitual por muchos grupos. La ETE puede cuantificar el volumen ventricular y medir débito cardiaco. Como se observó en este caso, existe buena correlación con la termodilución ${ }^{11-14}$. Esto, sin embargo, requiere un operador entrenado. Dada la velocidad de los cambios en el intraoperatorio, una evaluación cualitativa parecería suficiente para evaluar la función cardiaca. Esto además requiere grados de entrenamiento relativamente fáciles de alcanzar ${ }^{15}$.

El diagnóstico y corrección de la isquemia miocárdica durante el intraoperatorio es un hecho con implicancias pronósticas. La motilidad segmentaria de la pared ventricular no es fácil de evaluar, pero posee una alta sensibilidad ${ }^{16}$. Estudios humanos y animales muestran que la disminución del flujo coronario se acompaña rápidamente de aparición de alteraciones de esta, que preceden la aparición de alteraciones electrocardiográficas ${ }^{16-18}$. En cirugía aórtica y coronaria, la aparición de nuevas áreas de alteración de la motilidad ventricular se asoció a una mayor incidencia de infarto miocárdico en el postoperatorio ${ }^{19,20}$. La motilidad puede evaluarse visualizando una imágen transgástrica en eje corto, la cual es relativamente sencilla de obtener y sería la más indicada para el intraoperatorio ya que refleja la distribución de las tres arterias coronarias principales. En el paciente 3 , la normalidad en la motilidad ventricular en las distintas proyecciones permitió decidir realizar la cirugía, evitando postergaciones innecesarias, o realización de estudio adicional.

Nuestra experiencia con ETE en cirugía no cardiaca nos ha permitido evidenciar problemas frente a los cuales la monitorización habitual podría no ser suficiente para determinar el origen del deterioro de la situación hemodinámica, lo cual puede llevarnos a tomar decisiones que determinan mayor costo, retraso, e incluso deterioro de la condición del paciente. Por esto creemos importante incorporar la ETE como una herramienta diagnóstica en cirugía no cardiaca, ya que permite un diagnóstico más precoz y preciso de la alteración hemodinámica y su posible causa. Debe enfatizarse que, una incorrecta realización del exámen o interpretación de las imágenes, puede determinar complicaciones graves, por lo que al igual como fue con la electrocardiografía, el apoyo cardiológico en el entrenamiento de otros especialistas que requieran el uso de esta técnica resulta indispensable.

\section{Referencias}

1. Practice guidelines for perioperative transesophageal echocardiography. An updated report by the American Society of Anesthesiologists and the Society of Cardiovascular Anesthesiologists Task Force on Transesophageal Echocardiography. Anesthesiology; 112: 1084-96.

2. Fleisher LA, Beckman JA, Brown KA, Calkins H, Chaikof EL, Fleischmann KE, et al. ACC/AHA 2007 Guidelines on Perioperative Cardiovascular Evaluation and Care for Noncardiac Surgery: Executive Summary: A Report of the American College of Cardiology/American Heart Association Task Force on Practice Guidelines (Writing Committee to Revise the 2002 Guidelines on Perioperative Cardiovascular Evaluation for Noncardiac Surgery) Developed in Collaboration With the American Society of Echocardiography, American Society of Nuclear Cardiology, Heart Rhythm Society, Society of Cardiovascular Anesthesiologists, Society for Cardiovascular Angiography and Interventions, Society for Vascular Medicine and Biology, and Society for Vascular Surgery. J Am Coll Cardiol 2007; 50: 1707-32. 
3. Smith I, Benzie RJ, Gordon NL, Kelman GR, Swapp GH. Cardiovascular effects of peritoneal insufflation of carbon dioxide for laparoscopy. Br Med J 1971; 3: 410-1.

4. Hofer CK, Furrer L, Matter-Ensner S, Maloigne M, Klaghofer R, Genoni M, et al. Volumetric preload measurement by thermodilution: a comparison with transoesophageal echocardiography. Br J Anaesth 2005; 94: 748-55.

5. Darmon PL, Hillel Z, Mogtader A, Mindich B, Thys D. Cardiac output by transesophageal echocardiography using continuous-wave Doppler across the aortic valve. Anesthesiology 1994; 80: 796-805; discussion 25A.

6. Cunnion RE, Natanson C. Echocardiography, pulmonary artery catheterization, and radionuclide cineangiography in septic shock. Intensive Care Med 1994; 20 : 535-7.

7. Stephan F, Flahault A, Dieudonne N, Hollande J, Paillard F, Bonnet F. Clinical evaluation of circulating blood volume in critically ill patients-contribution of a clinical scoring system. Br J Anaesth 2001; 86: 754-62.

8. Michard F. Volume management using dynamic parameters: the good, the bad, and the ugly. Chest 2005; 128: 1902-3.

9. Mangano DT. Monitoring pulmonary arterial pressure in coronary-artery disease. Anesthesiology 1980; 53: 364-70.

10. Marik PE, Baram M, Vahid B. Does central venous pressure predict fluid responsiveness? A systematic review of the literature and the tale of seven mares. Chest 2008; 134: 172-8.

11. Lang RM, Bierig M, Devereux RB, Flachskampf FA, Foster E, Pellikka PA, et al. Recommendations for chamber quantification. Eur J Echocardiogr 2006; 7: 79-108.

12. Perrino AC, Jr., Harris SN, Luther MA. Intraoperative determination of cardiac output using multiplane transesophageal echocardiography: a comparison to thermodilution. Anesthesiology 1998; 89: 350-7.

13. Cheung AT, Savino JS, Weiss SJ, Aukburg SJ, Berlin JA.
Echocardiographic and hemodynamic indexes of left ventricular preload in patients with normal and abnormal ventricular function. Anesthesiology 1994; 81: 376-87.

14. Poelaert JI, Schupfer G. Hemodynamic monitoring utilizing transesophageal echocardiography: the relationships among pressure, flow, and function. Chest 2005; 127: 379-90.

15. Melamed R, Sprenkle MD, Ulstad VK, Herzog CA, Leatherman JW. Assessment of left ventricular function by intensivists using hand-held echocardiography. Chest 2009; 135: 1416-20.

16. Hauser AM, Gangadharan V, Ramos RG, Gordon S, Timmis GC. Sequence of mechanical, electrocardiographic and clinical effects of repeated coronary artery occlusion in human beings: echocardiographic observations during coronary angioplasty. J Am Coll Cardiol 1985; 5: 193-7.

17. Battler A, Froelicher VF, Gallagher KP, Kemper WS, Ross J. Dissociation between regional myocardial dysfunction and ECG changes during ischemia in the conscious dog. Circulation 1980; 62: 735-44.

18. Gallagher KP, Kumada T, Koziol JA, McKown MD, Kemper WS, et al. Significance of regional wall thickening abnormalities relative to transmural myocardial perfusion in anesthetized dogs. Circulation 1980; 62: 1266-74.

19. Roizen MF, Beaupre PN, Alpert RA, Kremer P, Cahalan MK, Shiller N, et al. Monitoring with two-dimensional transesophageal echocardiography. Comparison of myocardial function in patients undergoing supraceliac, suprarenal-infraceliac, or infrarenal aortic occlusion. J Vasc Surg 1984; 1: 300-5.

20. Smith JS, Cahalan MK, Benefiel DJ, Byrd BF, Lurz FW, Shapiro WA, et al. Intraoperative detection of myocardial ischemia in high-risk patients: electrocardiography versus two-dimensional transesophageal echocardiography. Circulation 1985; 72: 1015-21. 